ABSTRACTED/INDEXED IN Baidu Scholar · Cabell's Directory · Celdes · CNKI Scholar (China National Knowledge Infrastructure) · CNPIEC · De Gruyter: IBR (International Bibliography of Reviews of Scholarly Literature in the Humanities and Social Sciences); IBZ (International Bibliography of Periodical Literature in the Humanities and Social Sciences) · EBSCO (relevant databases) - EBSCO Discovery Service - Elsevier: SCOPUS - ERIH PLUS (European Reference Index for the Humanities and Social Sciences) · Genamics JournalSeek · Google Scholar · Informationszentrum für Fremdsprachenforschung (IFS) · J-Gate · JournalGuide · JournalTOCs · KESLI-NDSL (Korean National Discovery for Science Leaders) - Microsoft Academic · MLA International Bibliography · Naviga (Softweco) · Primo Central (ExLibris) · ProQuest (relevant databases) $\cdot$ ReadCube $\cdot$ ResearchGate · SCImago (SJR) - Sherpa/RoMEO - Summon (Serials Solutions/ProQuest) - TDNet · UB Frankfurt: BDSL Bibliographie der deutschen Sprach- und Literaturwissenschaft; BLL Bibliographie Linguistischer Literatur; OLC Linguistik · Ulrich's Periodicals Directory/ulrichsweb · WanFang Data - WorldCat (OCLC)

Preis- und Bezugsinformationen, Hinweise zur Manuskripteinrichtung und zu älteren Jahrgängen stehen unter www.degruyter.de/les zur Verfügung.

ISSN 0023-9909 • e-ISSN 1868-0267

VERANTWORTLICHE herAusgeber Prof. Dr. Hannelore Lee-Jahnke, Genf, Prof. Dr. Peter A. Schmitt, Leipzig

REDAKTION Doreen Schoon-Hammermann, Bernburg

JOURNAL MANAGER Anna Barkhoff, De Gruyter, Genthiner Straße 13, 10785 Berlin, Germany, Tel.: +49 (0)3026005 - 317, Fax: +49 (0)3026005 - 250,

E-Mail: anna.barkhoff@degruyter.com

ANZEIGENVERANTWORTLICHE Claudia Neumann, De Gruyter, Genthiner Straße 13, 10785 Berlin, Germany, Tel.: +49 (0)30 26005 - 226, Fax: +49 (0)30 26005 - 264, E-Mail: anzeigen@ degruyter.com

SATZ jürgen ullrich typosatz, Nördlingen

DRUCK Franz X. Stückle Druck und Verlag e.K., Ettenheim Printed in Germany

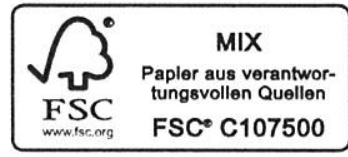


Federica Masiero*

\section{Die Übersetzung des Possessivpronomens dritter Person bei Bezugsambiguitäten: eine kontrastive Studie Deutsch / Italienisch}

https://doi.org/10.1515/les-2018-0008

Abstract: The present paper is concerned with the German adnominal demonstrative pronouns dessen und deren when these are used in place of the thirdperson possessive pronouns, in order to avoid ambiguities in resolution (Hans ${ }_{1}$ arbeitet mit Max $_{2}$ und seiner ${ }_{1 / 2}$ Frau / Hans ${ }_{1}$ arbeitet mit Max 2 und dessen 2 Frau 'Hans works with Max and his wife'). The central aim of the paper is to present and discuss the different possibilities available in Italian to translate German dessen und deren. The investigation is carried out on the basis of a corpus of literary texts.

Schlagwörter: dessen/deren, Demonstrativpronomina, Disambiguierung, Deutsch/Italienisch

\section{Auflösung von Bezugsambiguitäten des Possessivpronomens dritter Person im Deutschen}

Um eine Mehrdeutigkeit des Possessivpronomens dritter Person zu vermeiden, können im Deutschen die unveränderlichen Formen dessen und deren eingesetzt werden. Diese Formen gehören im heutigen Deutsch neben derer und denen zum Paradigma von der, die, das als Demonstrativ- und Relativpronomen. ${ }^{1}$ Beide Formen sind im Genitiv. Dessen ist Singular Maskulinum, deren ist Singular

1 Deren und derer sind beide Genitiv Singular Femininum und Genitiv Plural. Über ihre Verwendung herrscht eine gewisse Unsicherheit. Vgl. Bærenzen (1995; 2002), Eggers (1980), Engelen (1999), Najar (1996).

*Kontaktperson: Federica Masiero, Dipartimento di Studi linguistici e letterari, Via B. Pellegrino 26, I-35137 Padova, E-Mail: federica.masiero@unipd.it 
Femininum und Plural aller drei Genera. Unsere Aufmerksamkeit gilt hier dessen und deren als Demonstrativpronomen.

Die Verwendung von dessen und deren als Demonstrativpronomen ist insgesamt seltener belegt als die Verwendung als Relativpronomen. Dies beruht darauf, dass das Demonstrativum einer Konkurrenz durch das flektierbare Possessivum ihr- und sein- (s. 1-2) und unter Umständen durch die Genitivform ihrer und seiner des Personalpronomens (s. 3-5) unterliegt.

(1) Sie begrüßte ihren Freund und dessen (statt: seinen) Sohn.

(2) Er begrüßte seine alte Lehrerin und deren (statt: ihre) Tochter.

(3) Er hatte vor drei Jahren Max und Ewa kennen gelernt. Er erinnerte sich deren (statt: ihrer) nicht mehr.

(4) Er hatte vor drei Jahren Ewa kennen gelernt. Er erinnerte sich deren (statt: ihrer) nicht mehr.

(5) Er hatte vor drei Jahren Max kennen gelernt. Er erinnerte sich dessen (statt: seiner) nicht mehr.

Diese Konkurrenz besteht für das Relativum dessen und deren nicht, da das Possessivum und das Personalpronomen nicht als Relativpronomen auftreten können. Im Mittelpunkt der folgenden Untersuchung stehen dessen und deren als Demonstrativpronomen, und zwar, wenn sie adnominal verwendet werden, so wie (1) und (2).

Die geltenden Normen, wie sie im Duden (2009:§ 375) und mehr oder weniger übereinstimmend in den meisten Grammatiken des Deutschen formuliert sind, besagen, dass das Demonstrativpronomen häufig anstelle des entsprechenden Possessivpronomens gebraucht werden könne,

(6) Hans begrüßte seine Schwester und ihren/deren Mann.

und dass das Demonstrativpronomen dem Possessivpronomen vorzuziehen sei, wenn beim Possessivpronomen mehrere Bezüge möglich seien (u.a. Duden 1995:§ 560; Engel 1988:534; Helbig/Buscha 1998:328). Nach den meisten Grammatiken schaffen in solchen Fällen dessen und deren Klarheit, indem sie sich immer nur auf das letzte infrage kommende Substantiv beziehen, wobei bei dessen und deren ein Bezug auf das Subjekt ausgeschlossen ist. Während in (7a) der Bezug von dessen eindeutig ist,

(7a) Hans ging mit Max und dessen Frau spazieren.

kann der Satz

(7b) Hans ging mit Max und seiner Frau spazieren.

auf zweierlei Weise interpretiert werden: In (7b) kann sowohl Hans' Frau als auch Max' Frau die weibliche Begleitperson sein. 
Das Demonstrativpronomen lässt sich in diesem Gebrauch, wie oben angemerkt, nicht auf das Subjekt beziehen. In

(8) Hans fand dessen Auto.

kann es sich nur um das Auto einer anderen Person handeln, und nicht um das Auto von Hans. In

(9) Hans fand sein Auto.

kann es sich entweder um das Auto von Hans oder um dasjenige einer anderen Person handeln. Die Lesart ergibt sich aus dem Kontext.

Es sei auch erwähnt, dass sich dessen und deren schlecht auf ein Personalpronomen beziehen können. In

(10) Hans ging mit ihm und dessen Frau spazieren.

bezieht sich dessen bevorzugt auf eine andere Person als auf das Denotat von ihm.

Es gibt auch Grammatiken, die die Verwendung von dessen und deren anstelle des Possessivpronomens bei Bezugsambiguitäten nicht ansprechen. $\mathrm{Zu}$ erwähnen sind u.a. Zifonun/Hoffmann/Strecker (1997), Eisenberg (1989) sowie Hentschel (2010).

Unter den gängigen Grammatiken nimmt Weinrich (1993) eine Sonderstellung ein. Der Autor spricht von dessen und deren als Varianten der Possessivpronomina. Er behandelt sie nicht als Mittel zur Auflösung von Bezugsambiguitäten, sondern er sieht ihre Funktion darin, ein vorangegangenes Auffälligkeitssignal weiterzuführen. So ermögliche deren in einem Beispiel wie

(11) Sehen Sie die Dame dort? Mit deren Bruder bin ich oft ausgegangen.

im Unterschied zu dem entsprechenden Possessivpronomen die Verlängerung der im vorangehenden Satz vorhandenen Auffälligkeitsgeste, die das Possessivpronomen abbauen würde.

In den von den Grammatiken angeführten Beispielen scheint die Proximität das entscheidende Kriterium für die Resolution von dessen und deren als Demonstrativpronomen zu sein. Der Subjektstatus des potenziellen Antezedenten spielt ebenfalls eine wichtige Rolle, da er einen Bezug nicht erlaubt.

In der Forschung ist aber die Proximität als maßgeblicher Faktor für die Resolution von Demonstrativa umstritten (Zifonun 2005:102). Nach welchen Kriterien dessen und deren ihren Antezedenten wählen, ist nur unzureichend geklärt. So argumentieren Bosch/Katz/Umbach (2007) aufgrund von experimenteller Evidenz, dass bei der Auflösung der Demonstrativpronomina der, die, das ein Antezedent mit Subjektstatus bzw. Topikstatus vermieden werde und somit nicht die Abfolge der möglichen Antzedenten entscheidend sei. 
(12) Paul $_{\mathrm{i}}$ wollte mit Peter ${ }_{\mathrm{j}}$ laufen gehen. Aber $\mathrm{er}_{\mathrm{i} / \mathrm{j}} /$ der $_{\mathrm{*}_{\mathrm{i} / \mathrm{j}}}$ war leider erkältet.

In (12) bezieht sich der nicht auf das Subjekt bzw. nicht auf das Topik, da man annehmen kann, dass Paul Topik ist.

Die neuere Studie von Hinterwimmer/Bosch (im Ersch.) argumentiert wiederum, weder die grammatische Funktion noch der informationsstrukturelle Status des möglichen Antezedenten sei geeignet, die Resolution von Demonstrativa zu erklären. Hinterwimmer/Bosch (im Ersch.) führen den Begriff des Perspektivezentrums (perspectival centre) ein. Das Perspektivezentrum bezieht sich auf das Individuum, aus dessen Perspektive ein Ereignis bzw. eine Handlung geschildert oder bewertet wird. Sie argumentieren, dass ein Demonstrativum einen Antezedenten vermeide, der das aktuelle Perspektivezentrum des Satzes denotiert.

(13) $\quad$ Paul $_{\mathrm{i}}$ wollte mit Peter laufen gehen. $_{\mathrm{j}} \mathrm{r}_{\mathrm{i} / \mathrm{j}} /$ Der $_{\mathrm{i} / \mathrm{j}}$ sucht sich immer Leute als Trainingspartner aus, die nicht fit sind.

In (13) kann sich der auf Paul beziehen, obwohl Paul im ersten Satz als Subjekt und, wie anzunehmen ist, auch als Satztopik auftritt. Da aber in (13) der zweite Satz aus der Perspektive des Sprechers formuliert wird, ist Paul nicht das perspektivische Zentrum. Dies ist anders in (12). Hier ist das naheliegende perspektivische Zentrum des zweiten Satzes Paul. Dies wird durch die Tempusform, aber auch durch das Satzadverbial nahegelegt. Als Perspektivenzentrum ist Paul kein mögliches Denotat von der.

Obwohl die beiden Studien unterschiedliche Annahmen zur Resolution von Demonstrativpronomina liefern, ergibt sich aus den beiden Ansätzen, dass dessen und deren eine wichtige Funktion zukommen kann, wenn es darum geht, Bezugsambiguitäten zu vermeiden. Bei Bosch/Katz/Umbach (2007) ist das Topik im vorangehenden Satz das Element, das nicht für die Resolution des Demonstrativpronomens infrage kommt. Bei Hinterwimmer/Bosch (im Ersch.) ist es das Perspektivenzentrum des gegenwärtigen Satzes, auf das das Demonstrativpronomen nicht verweisen darf. Gibt es bezüglich der grammatischen Form im Prinzip mehrere mögliche Bezugselemente für ein Demonstrativpronomen, aber eines davon ist ein Topik bzw. ein Perspektivenzentrum, so fällt dieses als möglicher Referent weg. Dies wäre bei der Verwendung eines Personalpronomens nicht der Fall. Das Demonstrativpronomen kann deshalb häufig desambiguierende Funktion übernehmen.

Bosch/Katz/Umbach (2007) und Hinterwimmer/Bosch machen ihre jeweiligen Vorschläge nur für Demonstrativpronomen im Nominativ und Akkusativ. Es ist aber eine plausible Annahme, dass die entsprechenden Regularitäten auch für Demonstrativpronomen im Genitiv gelten. Diese Annahme soll im Folgenden gemacht werden, d.h., wir gehen davon aus, dass ein Demonstrativpro- 
nomen im Genitiv nicht auf ein Topik bzw. auf ein Perspektivenzentrum verweisen kann.

(14) Paul $_{\mathrm{i}}$ wollte mit Peter ${ }_{\mathrm{j}}$ laufen gehen. Aber seine $\mathrm{i}_{\mathrm{i} / \mathrm{j}}$ dessen $_{\mathrm{*}_{\mathrm{i} / \mathrm{j}}}$ Frau war leider nicht einverstanden.

Das Demonstrativpronomen im Genitiv kann demnach wie das Demonstrativpronomen im Nominativ oder Akkusativ eine desambiguierende Funktion aufweisen. Für unsere Korpusbeispiele wird es allerdings nicht erheblich sein, ob ein Demonstrativpronomen im Genitiv ein Topik oder ein Perspektivenzentrum vermeidet, da in unserem Beispielen das Topik des Vorsatzes und das Perspektivenzentrum des aktuellen Satzes auf denselben Referenten verweisen.

\section{Auflösung von Bezugsambiguitäten des Possessivpronomens dritter Person im Italienischen}

Bei Bezugsambiguitäten des Possessivpronomens dritter Person raten die berücksichtigten italienischen Grammatiken einstimmig zur Verwendung des Adjektivs propri- (= eigen) ${ }^{2} \quad$ (Serianni/Castelvecchi 1988:269; Renzi/Salvi/Cardinaletti 1988:628f; Brunet 1978:182-184; Schwarze 2009:33). Für diese Form gelten spezielle Verwendungsrestriktionen. Erstens kann sich propri- nur auf das Subjekt beziehen. In (15a)

(15a) Mario $_{\mathrm{i}}$ vide Carlo con $_{\mathrm{j}}$ sua $_{\mathrm{i} / \mathrm{j}}$ moglie.

(15b) Mario $_{\mathrm{i}}$ vide Carlo $_{\mathrm{j}}$ con la propria $\mathrm{i}_{\mathrm{i} /{ }_{\mathrm{j}}}$ moglie.

ist der Antezedent von sua zweideutig. sua könnte sich sowohl auf Carlo als auch auf Mario beziehen. Der Gebrauch der Form propria in (15b) verschafft Eindeutigkeit, indem propria unmissverständlich auf das Subjekt, Mario, Bezug nimmt. Zweitens muss sich der Antezedent von propri- im selben Teilsatz befinden, in dem propri- vorkommt.

(16a) Giorgio vuole che $\left[\mathrm{Silvia}_{\mathrm{i}}\right.$ riordini la propria ${ }_{\mathrm{i}}$ camera].

\footnotetext{
2 Die Form propri- tritt im Italienischen außer als Adjektiv auch als Adverb und dann mit der Bedeutung 'genau' auf (z.B.: proprio adesso = genau jetzt). Propri- als Adjektiv ist anstelle des Possessivpronomens obligatorisch, wenn das Subjekt unbestimmt ist (z.B.: si dorme bene nel proprio letto $=$ man schläft gut im eigenen Bett). Als Adjektiv kann propri- des Weiteren substantiviert werde (z.B.: vivere del proprio = für sich selber sorgen).
} 


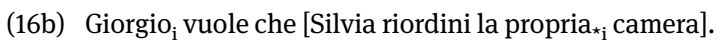

Bei Bezugsambiguitäten des Possessivpronomens dritter Person wird in den Grammatiken neben propri- die Benutzung der Präpositionalphrasen di lui/di lei (= von ihm, von ihr) empfohlen. Diese Formen sind einzusetzen, wenn sich das Possessivpronomen nicht auf das Subjekt bezieht, d.h., wenn die Verwendung von propri- nicht möglich ist (Serianni/Castelvecchi 1988:269; Renzi/Salvi/Cardinaletti 1988:628; Brunet 1978:186; Schwarze 2009:33). In (17) bewirkt der Ersatz von sua durch di lui

(17) Mario $_{\mathrm{i}}$ vide Carlo $_{\mathrm{j}}$ con la moglie di lui ${ }_{\mathrm{i}_{\mathrm{i}} \mathrm{j}}$.

einen eindeutigen Bezug auf Carlo.

Die Formen di lui/di lei müssen mit dem Genus des Antezedenten übereinstimmen. In der Gegenwartssprache werden sie dem Substantiv nachgestellt. Ihre Voranstellung (con la di lui moglie) ist für die Amts- und Literatursprache typisch (Serianni/Castelvecchi 1988:265; Brunet 1978:186-187; Schwarze 2009:33).

\section{Fragestellung und empirische Analyse}

Der vorliegende Beitrag hat einen sprachvergleichenden Ansatz. Mein primäres Ziel ist, anhand einer Analyse von authentischen Beispielen zu untersuchen, wie die adnominalen Demonstrativpronomen dessen und deren im Italienischen wiedergegeben werden, wenn sie $\mathrm{zu}$ einem eindeutigen anaphorischen Bezug im Quelltext führen. Dabei werden wir aber auch auf einige wenige Fälle stoßen, in denen die Verwendung von dessen und deren mit einer Bezugsambiguität einhergeht. Durch den Vergleich von Originaltexten und ihren Übersetzungen ins Italienische soll ermittelt werden, welche Varianten in der Übersetzungspraxis eingesetzt werden. Das Korpus, das hierfür ausgewertet wird, besteht aus literarischen Texten (s. Quellenkorpus). Durch die Begrenzung auf fiktionale narrative Texte bleibt das Korpus weitgehend einheitlich. Insgesamt werden der Untersuchung Werke aus den 20er bis zu den 60er Jahren des 20. Jahrhunderts zugrunde gelegt. Die relativ breite Spanne meines Korpus ist nicht relevant, da das Phänomen in dem analysierten Zeitabschnitt diachron keine Veränderungen erfährt.

Anhand des Korpus wurden insgesamt 130 deutsche Belege zusammengestellt. ${ }^{3}$ Alle Belege weisen die Form dessen oder deren als adnominale Demons-

3 Wie zu erwarten war, ist die Verwendung von dessen und deren als Relativpronomen in unserem Korpus viel häufiger belegt als die als Demonstrativpronomen. 
trativpronomen auf. Dessen und deren werden in allen Belegen verwendet, um eine Mehrdeutigkeit des Pronomens dritter Person zu vermeiden. Die Belege, in denen dessen und deren als reine Variante des entsprechenden Possessivpronomens vorkommen, (s. 6), werden nicht berücksichtigt:

(18) oder wenn sie, gelegentlich allein im Kinderzimmer zurückbleibend, unerwünschte Muße hatte, über ihr Los nachzudenken, da ward ihr dessen ganze Kläglichkeit wie in einer plötzlichen Erleuchtung klar (Therese, 25)

Für eine bessere Übersichtlichkeit werden die Belege in vier Gruppen aufgeteilt. Entscheidend für die Gruppierung der Belege ist die Stellung von dessen und deren gegenüber ihren Antezedenten. Für jede Gruppe werden einige repräsentative Belege mit ihrer italienischen Übersetzung präsentiert.

\section{Gruppe}

Die Belege dieser Gruppe zeichnen sich dadurch aus, dass sich die adnominale Demonstrativform nicht im selben Satz wie ihr Antezedent befindet. Diese Gruppe umfasst 81 der insgesamt 130 Belege. Bei vier Belegen werden die adnominalen Demonstrativformen ins Italienische nicht übersetzt. Für unsere Analyse sind sie deshalb nicht relevant. Im Folgenden werden die verbleibenden 77 Belege in vier Untergruppen präsentiert. Die Untergruppen unterscheiden sich dadurch, mit welcher Form die Demonstrativpronomina ins Italienische übersetzt werden.

a) In 60 der 77 Belege werden die Formen dessen und deren durch das Possessivpronomen übersetzt. Durch die Verwendung des Possessivpronomens ergibt sich im Italienischen rein grammatisch betrachtet eine Bezugsambiguität, die im Original nicht vorhanden ist. Die Disambiguierung erfolgt durch den Kontext.

(19a) und [da] niemand nach dem ertrunkenen Wranka ${ }_{i}$ peinliche Fragen stellte, kroch Koljaiczek, der die ähnliche Statur und den gleichen Rundschädel wie der Ertrunkene

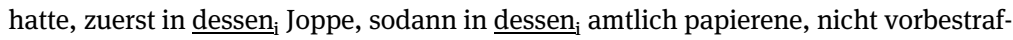
te Haut (Blechtrommel, 11)

(19b) e [poiché] a nessuno venne in mente di far domande imbarazzanti sull'annegato Wranka $\mathrm{i}_{\mathrm{i}}$, Koljaiczek $\mathrm{k}_{\mathrm{j}}$ che aveva quasi la stessa statura e lo stesso cranio tondeggiante,

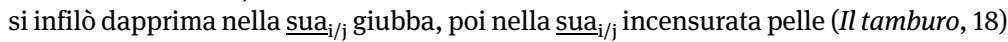

(20a) [...] wandte er [...] den Kopf über die linke Schulter gegen den Platz seines Liebhabers $_{\mathrm{i} .}$ Er fand nicht dessen ${ }_{\mathrm{i}}$ Augen (Tod in Venedig, 164)

(20b) e [Tadzio] gettava uno sguardo al di sopra della spalla sinistra verso il suo amatore . $^{\text {. }}$ [Lui] $]_{\mathrm{j}}$ non incontrava i $\underline{\mathrm{suoi}}_{\mathrm{i} / \mathrm{j}}$ occhi (Morte a Venezia, 165) 
(21a) Aber wenn diese Cowboys, Goldgräber, Trapper dann hinter den Mädchen ${ }_{i}$ hergehen und mit auf $\underline{\text { deren }}_{\mathrm{i}}$ Zimmer wollen, kriegen sie meistens die Tür vor der Nase zugeknallt. (Ansichten, 119)

(21b) ma quando poi [i cowboys, i cercatori d'oro e i cacciatori $]_{j}$ vogliono andare dietro alle ragazze $_{\mathrm{i}}$ e salire nelle $\underline{\text { loro }}_{\mathrm{i} / \mathrm{j}}$ camere, si vedono por lo più sbattere la porta in faccia. (Opinioni, 99)

Die italienischen Possessivformen sua in (19b), suo in (20b) und loro in (21b) sind rein grammatisch zweideutig. Die Form di lui, die in (19b) und (20b) die Ambiguität auflösen könnte ((19b): la giubba di lui/la pelle incensurata di lui; (20b): lo sguardo di lui), wird nicht eingesetzt. In (21b) würde auch die Präpositionalphrase di loro, die die Grammatiken empfehlen, die Ambiguität nicht auflösen, da sie im Unterschied zu di lui kein Genus festlegt. Die Festlegung des Genus ist hier entscheidend für die Disambiguierung, da alle potenziellen Antezedenten im Plural sind. Der Bezug könnte aber durch die Verwendung einer Präpositionalphrase mit einem Demonstrativum wie quest- (dies-) klargestellt werden (Serianni/Castelvecchi 1988:266). Durch di queste (Fem. Pl.) wird sowohl das Genus als auch den Numerus bestimmt. Diese Form wird ebenfalls nicht verwendet.

b) Sieben der 77 Belege weisen die Form deren auf, die im Italienischen durch das Possessivpronomen loro dritter Person Plural wiedergegeben wird, ohne - im Unterschied zu (21b) - eine Bezugsambiguität zu ergeben. Die Form loro erweist sich in diesen Belegen als eindeutig im Gegensatz zu der Form deren, die in den Belegen rein grammatisch ambig ist.

(22a) Er war der einzige junge Verteidiger, die anderen waren alt, einige, wie sich bald zeigte, [alte Nazis] $]_{i}$. Hannas ${ }_{j}$ Verteidiger vermied deren ${ }_{i / j}$ Jargon $($ Vorleser, 92)

(22b) Era l'unico difensore giovane, gli altri erano tutti anziani, e alcuni, come si vide ben presto, dei vecchi nazisti $i_{i}$. Il difensore di Hanna evitava il $\underline{\text { loro }}_{i}$ gergo (A voce alta, 79)

In (22b) stellt das italienische Possessivpronomen loro dritter Person Plural einen eindeutigen Bezug her, da es die anderen potenziellen Antezedenten, die alle im Singular stehen, ausschließt. In der deutschen Version wäre auch die loro entsprechende Form ihre ambig: Sie könnte sich in (22a) rein grammatisch sowohl auf Hanna als auch auf alte Nazis beziehen. Dies hängt mit den Stammverschiedenheiten im Paradigma des Possessivpronomens dritter Person Plural in den beiden Sprachen zusammen. Im Deutschen ist der Femininum-Stamm ihr-gleichzeitig Singular- und Pluralstamm.

c) Fünf der 77 Belege weisen in der italienischen Übersetzung zur Wiedergabe von dessen und deren die Form ne auf.

(23a) [der Fürst] erklärte, daß er bereits einhundert Mann und einen Heerhaufen ${ }_{\mathrm{i}}$ zusam- $^{-}$ menzöge, und sich selbst an $\underline{\text { dessen }}_{i}$ Spitze setzen würde (Radetzkymarsch, 43) 
(23b) [il principe] dichiarò che stava raccogliendo cento uomini $i_{j}$ e un esercito ${ }_{i}$ e che $\underline{n e}_{i / j}$ avrebbe preso personalmente la testa (La marcia, 48)

Die Form ne entspricht in diesen Fällen einem Possessivpronomen dritter Person und wäre in (23b) auch durch die Präpositionalphrase di esso ersetzbar. ${ }^{4}$ Wie die Präpositionalphrasen kann ne nur anstelle eines Possessivpronomens eingesetzt werden, wenn der Antezedent nicht im selben Satz wie das Possessivpronomen steht. Im Unterschied zu der Präpositionalphrase di esso legt ne das Genus und den Numerus des Antezedenten nicht fest (Serianni/Castelvecchi 1988:253). Die Resolution von ne in (23b) ist wegen dieser Unterbestimmtheit ambig.

d) In fünf Fällen werden die deutschen Demonstrativpronomen durch das klitische Personalpronomen im Dativ gli (= ihm) wiedergegeben, das den Possessor bezeichnet.

(24a) Wenn man ihm [dem Heizer] den Schubal $_{i}$ hingehalten hätte, hätte er wohl $\underline{\text { dessen }}_{i}$ gehaßten Schädel mit den Fäusten aufklopfen können. (Amerika, 30)

(24b) Se gli si fosse abbandonato lo Schubal $_{\mathrm{i}}$, certo sarebbe stato capace di spaccargli ${ }_{i}$ coi pugni quella testa odiosa. (America, 70)

(25a) Er ging langsam in solchen Gedanken zum Heizer ${ }_{\mathrm{i}}$, zog dessen ${ }_{\mathrm{i}}$ Hand aus dem Gürtel (Amerika, 43)

(25b) E mentre seguiva queste idee si avvicinò lentamente al fochista ${ }_{\mathrm{i}}$, gli $_{\mathrm{i}}$ tolse la mano dalla cintura (America, 82)

Der Einsatz eines Klitikums im Dativ zum Ausdruck des Possessors ist bei Körperteilen, wie es in unseren Belegen der Fall ist, und generell bei unveräußerlichen Possessa möglich. Dies geschieht durch die sogenannte Konstruktion mit externem Possessor. Die entsprechende Konstruktion ist auch im Deutschen möglich.

(25c) ging in solchen Gedanken zum Heizer, zog ihm die Hand aus dem Gürtel.

In diesen Fällen ermöglicht der Einsatz der Konstruktion mit externem Possessor im Italienischen (25b) die Disambiguierung des Bezuges, indem keine Resolution mit dem Subjekt des Satzes möglich ist.

$4 \mathrm{Ne}$ tritt im Italienischen ferner als Lokaladverb (ne riparti domani = er ging morgen wieder los) und als Partikel in Verbindung mit Verben (se ne sta seduto con le mani in mano = er sitzt und bleibt dabei untätig). In beiden Verwendungen hat ne oft keine Entsprechung im Deutschen. 


\section{Gruppe}

Die zweite Gruppe enthält Belege, in denen dessen und deren im selben Satz wie ihr Antezedent stehen. Es handelt sich um 24 Belege. Bei drei werden die Demonstrativformen ins Italienische nicht übersetzt. Diese Belege sind für unsere Analyse nicht relevant. Die Ergebnisse werden in zwei Untergruppen präsentiert. Die Untergruppen unterscheiden sich dadurch, mit welcher Form die Demonstrativpronomina ins Italienische übersetzt werden.

a) In 18 Belegen werden dessen und deren durch das Possessivpronomen wiedergegeben. Wegen der Verwendung des Possessivpronomens ist in der italienischen Version der korrekte Bezug mithilfe des Kontexts zu ermitteln. Die Formen di questi (26b) und di lui (27b und 28b), die den Bezug disambiguieren könnten, werden nicht eingesetzt.

(26a) Aber er [der Verteidiger] war von einem hastigen Eifer, der seiner Mandantin ebenso

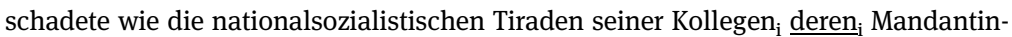
nen. (Vorleser, 92)

(26b) Ma era di uno zelo precipitoso e brusco, che nuoceva alla sua cliente tanto quanto [le tirate naziste $]_{\mathrm{j}}$ dei suoi colleghi $\mathrm{i}_{\mathrm{i}}$ danneggiavano le $\underline{\text { loroi }}_{\mathrm{j} \mathrm{j}}$ mandanti. $($ A voce alta, 79$)$

(27a) und es verwunderte ihn [Trotta], daß er in diesem Augenblick an den toten Max Demant $_{\mathrm{i}}$ denken mußte und an dessen $_{\mathrm{i}}$ Großvater (Radetzymarsch, 140)

(27b) e [Trotta $]_{j}$ fu estremamente sorpreso che in quell'istante gli venisse da pensare al povero Max Demant ${ }_{\mathrm{i}}$ e $\underline{\mathrm{suo}}_{\mathrm{i} / \mathrm{j}}$ nonno (La marcia, 334)

(28a) Im nächsten Frühjahr schon war die [Therese] genötigt, ihren Buben aus dem Hause des Schneidermeisters ${ }_{i}$ zu entfernen, weil er sich mit dessen ${ }_{i}$ Sohn [...] durchaus nicht zu vertragen schien. (Therese, 82)

(28b) La primavera dopo [Teresa $]_{\mathrm{j}}$ fu costretta ad allontanare il suo ragazzo dalla casa del sarto $_{\mathrm{i}}$ perché $[. .$.$] non sembrava proprio andare d'accordo con \underline{\mathrm{suo}}_{\mathrm{i} / \mathrm{j}}$ figlio $($ Teres $a, 93)$

b) In den übrigen drei Belegen wird deren durch die in den Grammatiken empfohlene Form di lei wiedergegeben, die den Bezug eindeutig macht.

(29a) während Herr Ruhwiedel den Besucher als ziemlich jungen Schlacks bezeichnete, der

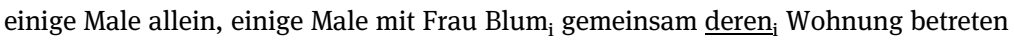
habe. (Blum, 32)

(29b) mentre il signor Ruhwiedel definiva il visitatore uno spilungone abbastanza giovane ch'era entrato, a volte da solo, a volte in compagnia della Blum ${ }_{\mathrm{i}}$, in casa di lei (Blum, 19.) 


\section{Gruppe}

Die dritte Gruppe umfasst 13 Belege, in denen die Demonstrativformen und ihr Antezedent zum selben Satzglied gehören. Das Satzglied besteht aus zwei koordinierten Nominalphrasen. Die Belege werden in zwei Untergruppen präsentiert.

a) In sieben Belegen wird das Demonstrativpronomen durch den unbestimmten Artikel übersetzt. Die Entscheidung für den unbestimmten Artikel zur Wiedergabe von dessen bzw. deren rührt daher, dass im Italienischen bei Verwandtschaftsbeziehungen die Zugehörigkeit oft nicht durch ein Possessivum explizit gemacht wird, es genügt der bestimmte oder der unbestimmte Artikel (Renzi/ Salvi/Cardinaletti 1988:381; Schwarze 2009:35). Der Kontext disambiguiert im Italienischen den Bezug.

(30a) Unter ihr, in drei Zimmern, von denen aber nur zwei benutzt wurden, wohnten ihre Mutter $_{\mathrm{i}}$ und deren ${ }_{i}$ ältere Schwester (Die Angst, 24)

(30b) Sotto di lei, in tre stanze di cui però soltanto due venivano utilizzate, abitavano sua madre con una sorella maggiore. (Prima del calcio, 35)

Durch die Verwendung des unbestimmten Artikels wird in der italienischen Version nicht erfasst, dass es sich um die einzige ältere Schwester handelt und nicht möglicherweise um eine unter mehreren.

b) In sechs Belegen findet sich ein zweideutiges Possessivpronomen. Hier könnte die Verwendung von propri in (31b) und von di lui in (32b) den Bezug klarstellen:

(31a) Nie hörte ich, was er [Mahlke] dachte. Am Ende bleiben sein Hals $_{i}$ und $\underline{\text { dessen }}_{i}$ viele Gegengewichte. (Katz und Maus, 31)

(31b) Non sentii mai quello che [lui $]_{j}$ pensava. Per cui, per finire, rimane soltanto la sua gola $_{\mathrm{i}}$, e i $\underline{\text { suoi }}_{\mathrm{ijj}}$ numerosi contrappesi. (Gatto e topo, 46)

(32a) Oskar übte Askese, magerte ab, wurde dem Dr. Hollatz $\mathrm{i}_{\mathrm{i}}$ und dessen ${ }_{\mathrm{i}}$ immer knochiger werdenden Assistentin Schwester Inge vorgeführt. (Blechtrommel, 96)

(32b) E [Oskar $]_{j}$ venne condotto dal dottor Hollatz $\mathrm{i}_{\mathrm{i}}$ e dalla $\underline{\text { sua }}_{\mathrm{i} / \mathrm{j}}$ assistente, l'infermiera Inge, che si faceva sempre più ossuta. (Il tamburo, 203)

\section{Gruppe}

Die vierte Gruppe besteht aus 12 Belegen. In diesen Belegen verweisen dessen und deren auf den letzten in einer Reihe möglicher Antezedenten. Der Antezedent gehört zum selben Satzglied wie dessen und deren. Im Unterschied zur 
dritten Gruppe besteht das Satzglied in dieser Gruppe aus mehr als zwei Nominalphrasen. Die Disambiguierung kommt in diesem Fall über die topologische Eigenschaft zustande: Dessen und deren verweisen eindeutig auf das Letztgenannte. In diesen Fällen genügt die Annahme, dass die Proximität der entscheidende Faktor für ihre Resolution ist. In dieser Funktion haben dessen und deren im Italienischen ihre Entsprechung in der Form quest'ultim-, die die Übersetzer auch einsetzen (Veldre/Gerner 2012:138). Sie verweist eindeutig auf das Letztgenannte.

(33a) Beweismittel für den ersten Hauptanklagepunkt waren ausschließlich das Zeugnis

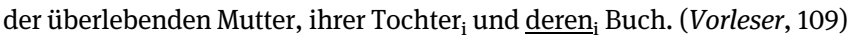

(33b) I soli elementi probatori a carico del primo principale capo d'accusa erano le testimonianze della madre e della figlia ${ }_{\mathrm{i}}$ sopravvissute, e il libro di quest'ultima ${ }_{\mathrm{i}}$. (A voce alta, 94)

(34a) und [Hedwig Bronski] brachte zur Taufe meines Sohnes Kurt ihren Ortsbauernführer,

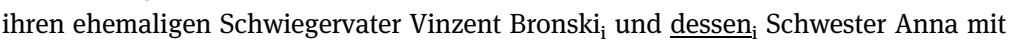
(Blechtrommel, 142)

(34b) e al battesimo di mio figlio Hedwig Bronski condusse il suo capo dei contadini, l'ex suocero Vinzent Bronski $\mathrm{i}_{\mathrm{i}}$ e la sorella di quest'ultimo ${ }_{\mathrm{i}}$. (Il tamburo, 209)

In (33b) könnte z.B. das Possessivpronomen suo (suo libro) sowohl auf madre als auch auf figlia bezogen werden. Beide Zuordnungen würden eine sinnvolle Aussage ergeben. Der Kontext würde hier keinen festen Anhaltspunkt bieten, der dem Leser / Hörer erlaubte, sich zwischen den beiden Optionen zu entscheiden.

\section{Kommentare zu den einzelnen Gruppen}

\section{Gruppe}

In 59 Belegen $(77 \%)$ entscheiden sich die Übersetzer bei der Wiedergabe von dessen und deren für die Possessivpronomen. Dadurch ergibt sich rein grammatisch in den meisten Fällen eine Bezugsambiguität, die im Original nicht vorhanden ist. Angesichts der grammatikalischen Ambiguität der Formen spielt die Suche nach einem Antezedenten im Kontext, der eine plausible Interpretation ergibt, eine entscheidende Rolle. In der Regel erfolgt dies problemlos.

Nur in sieben Belegen entscheiden sich die Übersetzer für ein Possessivpronomen, und zwar für loro dritter Person Plural, bei dem sich im Gegensatz zu den oben geschilderten Fällen keine Ambiguität ergibt. Die Eindeutigkeit folgt aus dem Paradigma des italienischen Possessivpronomens. Im Gegensatz zu dem deutschen Paradigma weist das italienische Paradigma in der dritten Person Plural die unflektierbare Form loro auf, die eindeutig auf ein Bezugswort im 
Plural verweist. Die entsprechende deutsche Form ihr- kann sowohl auf ein Bezugswort im Singular als auch im Plural verweisen.

Eine Bezugsambiguität, die im Deutschen rein grammatisch nicht vorhanden ist, ergibt sich des Weiteren im Italienischen aus der Übersetzung von dessen und deren durch die Form ne. Obwohl die Verwendung von ne verschiedenen Restriktionen unterliegt, die z.B. im selben Satz auftretende, potentielle Antezedenten ausschließen, trägt sie in unseren Belegen nicht zur Disambiguierung bei. Der Antezedent wird aber trotzdem aufgrund des Strebens nach einer plausiblen Aussage ohne Probleme identifiziert.

In fünf Fällen greifen die italienischen Übersetzer durch die Verwendung eines Klitikums auf die sogenannte Konstruktion mit externem Possessor zu. Das Verwendungsspektrum dieser Konstruktion ist eingeschränkt gegenüber der mit internem Possessor. Es handelt es sich meistens um die inalienable Relation zwischen Person und Körperteil wie im Fall unserer Belege. Konstruktionen mit externem Possessor schließen die pleonastische Setzung des adnominalen Possessivpronomens aus. Das klitische Pronomen ermöglicht einen eindeutigen Bezug.

\section{Gruppe}

Die Untersuchung der zweiten Gruppe bestätigt die Tendenz, die schon bei der ersten Gruppe festgestellt wurde. In 18 von den für unsere Analyse relevanten 21 Belegen entsteht im Italienischen durch die Verwendung des Possessivpronomens eine Bezugsambiguität, die im Original nicht vorhanden ist. Wiederum erfolgt die Disambiguierung durch den Kontext. Im Unterschied zu der ersten Gruppe befindet sich in dieser Gruppe der Antezedent im selben Satz. Die disambiguierende Form di lei wird nur in drei von den 21 Belegen eingesetzt. In dieser Gruppe ist der Einsatz der Form ne, die in der ersten Gruppe belegt ist, nicht möglich, da ne keinen Antezedenten im selben Satz erlaubt.

\section{Gruppe}

Von den zwei Untergruppen, aus denen diese Gruppe besteht, ist vornehmlich die erste interessant. Hier wird die Form deren durch den unbestimmten Artikel im Italienischen wiedergegeben, da sich die beiden Sprachen bei der Angabe von Verwandtschaftsbeziehungen anders verhalten: Im Italienischen wird viel häufiger als im Deutschen der bestimmte oder unbestimmte Artikel verwendet. Es ergibt sich keine grammatisch determinierte Zuordnung. In der zweiten Unter- 
gruppe verzichtet der Übersetzer durch die Verwendung des Possessivpronomens abermals auf eine eindeutige Resolution, die die Form propri ermöglicht hätte.

\section{Gruppe}

In dieser Gruppe kommt dessen und deren die spezifische Funktion zu, den topologisch nächsten Antezedenten aus mehreren potentiellen Antezedenten zu selektieren. Sie erfüllen eine strikt lokalisierende Funktion: Der Antezedent wird ausschließlich über seine topologisch-positionelle Eigenschaft im Text identifiziert. Die entsprechende Funktion wird im Italienischen durch quest'ultimo erfüllt, das eindeutig auf das Letztgenannte verweist und die von den Übersetzern eingesetzt wird. Die Übersetzung von dessen und deren durch ein Possessivpronomen würde zu einer Ambiguität führen, die der Kontext nicht auflösen könnte.

\section{Schlussfolgerungen}

Auf der Basis des untersuchten Korpus lassen sich bezüglich der Wiedergabe von dessen und deren in literarischen Texten folgende fünf Sachverhalte feststellen:

1. Die Übersetzer greifen bei literarischen Texten viel seltener auf grammatische Mittel zur Disambiguierung der Possessivrelation zurück, als es die Autoren der deutschen Quelltexte tun. In den italienischen Übersetzungen wird in $74 \%$ der Fälle auf den eindeutigen grammatischen Bezug, den die adnominalen Demonstrativpronomina dessen und deren in der deutschen Vorlage aufweisen, verzichtet.

2. Dabei bevorzugen die Übersetzer die Possessivpronomina und die Form ne, die rein grammatisch keinen eindeutigen Bezug ermöglichen, wobei der Gebrauch von ne weniger Verwendung findet als die Possessivpronomina, da ne bestimmte Verwendungsrestriktionen unterliegt.

3. In wenigen Fällen jedoch führt der Einsatz von Possessivpronomen im Italienischen zu einer Eindeutigkeit des Bezugs, die bei dessen und deren in der deutschen Vorlage nicht gegeben ist. Dies ergibt sich aufgrund von Unterschieden im Paradigma der Possessivpronomen der beiden Sprachen. Insbesondere hat sich gezeigt, dass die dritte Person Plural des italienischen Possessivpronomens (loro) zu einem eindeutigen Bezug führen kann.

4. Vereinzelt verzichten die Übersetzer auch ganz auf die Wiedergabe der anaphorischen Bedeutung von dessen und deren. Dies geschieht bei der Übersetzung von dessen und deren bei Verwandtschaftsbezeichnungen durch den unbestimmten Artikel, wobei ein Informationsverlust einhergeht. 
5. Zur Wiedergabe von dessen und deren kann ferner in der italienischen Sprache die Konstruktion mit externem Possessor eingesetzt werden. Durch den Ausdruck des Possessors durch ein Klitikum wird der Bezug klargestellt. Die Konstruktion mit externem Possessor unterliegt in beiden Sprachen bestimmten, teilweise verschiedenen Gebrauchsrestriktionen. Geht es um einen Bezug auf Körperteile, ist die Konstruktion in beiden Sprachen möglich.

Der quantitativ am häufigsten auftretende Fall wurde unter 1. dargestellt. Hier wurde herausgearbeitet, dass die von den Grammatiken empfohlenen Formen di lui / di lei und propri- von den Übersetzern kaum eingesetzt werden. Laut den Grammatiken ergänzen die genannten Formen das Paradigma der Possessivpronomen, indem sie bei Bezugsambiguitäten zu einer eindeutigen Interpretation führen können. In dieser Hinsicht kann man sie als die italienische Entsprechung von dessen und deren auffassen. Bei di lei und di lui, deren Einsatz bei unseren Belegen am häufigsten $\mathrm{zu}$ einer Disambiguierung führen könnten, handelt es sich - im Gegensatz zu den deutschen Formen dessen und deren - um postnominale Formen. Man kann vermuten, dass die Übersetzer di lui und di lei vermeiden wollen, weil sie sie als stilistisch wenig elegant empfinden. Dasselbe kann nicht von der Form propr- behauptet werden, die wie dessen und deren pränominal ist, die aber ebenfalls nicht benutzt wird. Der Verzicht auf den Einsatz der grammatischen Mittel, die zu einer Disambiguierung führen können, hat zur Folge, dass der Leser / Hörer des italienischen Textes eine stärkere interreferenzielle Leistung erbringen muss als der Leser des deutschen Ausgangtextes. Der Leser / Hörer des italienischen Textes muss nach einem passenden Antezedenten im Kontext suchen, der eine inhaltlich sinnvolle Aussage ermöglicht. Dem Kontext wird die Funktion überlassen, den Bezug klar zu machen, wobei dies in den von mir untersuchten Korpusbelegen durchaus problemlos möglich ist. Die Übersetzer nutzen nur dann die grammatischen Mittel der italienischen Sprache für einen eindeutigen Bezug, wenn der Kontext keine Stütze für eine Disambiguierung liefert. Die Autoren der Quelltexte benutzen hingegen die Form dessen und deren unabhängig davon, ob der Kontext hinreichende Hinweise für eine Disambiguierung aufweist. Damit unterstreichen sie, dass der Referent von dessen und deren ungleich dem Perspektivenzentrum ist.

\section{Quellenkorpus}

Böll, Heinrich (1963): Ansichten eines Clowns. Köln/Berlin: Kiepenheuer, 1970.

Böll, Heinrich (1965): Opinioni di un clown. Übersetzung von Amina Pandolfi. Milano: A. Mondadori. 
Böll, Heinrich (1974): Die verlorene Ehre der Katharina Blum. München: Taschenbuch Verlag. Böll, Heinrich (1975): L'onore perduto di Katharina Blum. Übersetzung von Italo Alighiero Chiusano. Torino: Einaudi.

Grass, Günther (1959): Die Blechtrommel. Darmstadt und Neuwied: Luchterhand, 1979. Grass, Günther (1962): Il tamburo di latta. Übersetzung von Lia Secci. Milano: Feltrinelli. Grass, Günther (1961): Katz und Maus. Darmstadt und Neuwied: Luchterhand, 1974.

Grass, Günther (1994): Gatto e topo. Übersetzung von Enrico Filippini. Milano: Feltrinelli. Handke, Peter (1970): Die Angst des Tormannes beim Elfmeter. München: Taschenbuch Verlag. Handke, Peter (1982): Prima del calcio di rigore. Übersetzung von Bruna Bianchi. Milano: Feltrinelli.

Kafka, Franz (1925): Der Prozeß. Frankfurt a.M.: Fischer, 1979.

Kafka, Franz (1976): /l processo. Übersetzung von Ervino Pocar. Milano: Mondadori. Kafka, Franz (1927): Amerika. Frankfurt a. M.: Fischer, 1946.

Kafka, Franz (1989): America. Übersetzung von Alberto Spaini. Milano: Mondadori.

Mann, Thomas (1912): Der Tod in Venedig / La morte a Venezia. Torino: Einaudi, 2004.

Roth, Joseph (1932): Radetzkymarsch. Köln: Kiepenheuer \& Witsch, 2014.

Roth, Joseph (1987): La marcia di Radetzky. Übersetzung von Laura Terreni e Luciano Foà. Milano: Adelphi.

Schlink, Bernhard (1995): Der Vorleser. Zürich: Diogenes.

Schlink, Bernhard (1998): A voce alta. Übersetzung von Rolando Zorzi. Firenze: Nuova Biblioteca Garzanti.

Schnitzler, Arthur (1926): Traumnovelle. Stuttgart: Philipp Reclam, 1994.

Schnitzler, Arthur (1997): Doppio sogno. Übersetzung von Giuseppe Farese. Milano: Adelphi.

Schnitzler, Arthur (1928): Therese. Chronik eines Frauenlebens. Frankfurt a. M.: Fischer Verlag, 2014.

Schnitzler, Arthur (1929): Teresa: cronaca d'una vita di donna. Übersetzung von B. GiacchettiSorteni. Milano: Modernissima.

\section{Literaturverzeichnis}

Bærentzen, Per (1995): "Zum Gebrauch der Pronominalformen deren und derer im heutigen Deutsch”. Beiträge zur Geschichte der deutschen Sprache 117/2, 199-217.

Bærentzen, Per. (2002): “Deren oder derer? Versuch einer ehrenrettenden Richtigstellung”. Beiträge zur Geschichte der deutschen Sprache 124/1, 144-147.

Brunet, Jacquelin (1978): Grammaire critique de l'italien. Paris: I'Université de Paris VIII.

Bosch, Peter / Katz, Graham / Umbach, Carla (2007): “The Non-Subject Bias of German Demonstrative Pronouns”. Monika, Schwarz-Friesel / Mareile, Consten / Manfred, Knees (2007) (eds): Anaphors in Text. Amsterdam: John Benjamins Publishing Company, 145-164.

Bosch, Peter / Hinterwimmer, Stefan (im Ersch.): Demonstrative Pronouns and Perspective.

Duden - Grammatik (2009). Der Duden in 12 Bänden. Bd. 4. Die Grammatik. 8., überarb. Auflage. Mannheim u.a.: Dudenverlag.

Duden - Grammatik (1995). Der Duden in 12 Bänden. Bd. 4. Die Grammatik. 5., völlig neu bearbeitete und erweiterte Auflage. Mannheim u.a.: Dudenverlag.

Eggers, Hans (1980): “Derer oder deren? Zur Normenproblematik im Deutschen”. Moderna språk $74 / 2,133-138$. 
Eisenberg, Peter (1989): Grundriss der deutschen Sprache. 2. überarbeitete und erweiterte Auflage. Stuttgart: Metzler.

Engel, Ulrich (1988): Deutsche Grammatik. Heidelberg: Groos.

Engelen, Bernhard (1999): "Derer oder deren? Zu einer Normunsicherheit und ihrer Geschichte". Freundenberg-Findeisen, Renate (1999) (Hrsg.): Ausdrucksgrammatik versus Inhaltsgrammatik. München: ludicium, 89-109.

Helbig, Gerard / Buscha, Joachim (1998): Deutsche Grammatik. Leipzig u.a.: Langenscheidt. Hentschel, Elke (2010) (Hrsg.): Deutsche Grammatik. Berlin / New York: de Gruyter.

König, Ekkehard / Haspelmath, Martin (1998): “Les constructions à possesseur externe dans les langues d'Europe”. Feuillet, Jack (1998) (Hrsg.): Actance et valence dans les languages de l'Europe. Berlin / New York: de Gruyter, 525-606.

Najar, Margarete (1996): “Ist die Unterscheidung von deren : derer ins Schleudern geraten?”. Pérennec, Marie-Hélène (1996) (Hrsg.): Pro-Formen des Deutschen. Tübingen: Stauffenburg-Verlag, 111-121.

Renzi, Lorenzo / Salvi, Giampaolo / Cardinaletti, Anna (1988): Grande grammatica di consultazione. Vol. III: Tipi di frase, deissi, formazione delle parole. Bologna: Il Mulino.

Schwarze, Christoph (2009): Grammatica della lingua italiana. Firenze: Carrocci.

Serianni, Luca / Castelvecchi, Alberto (1988): Grande grammatica italiana: italiano comune e lingua letteraria. Torino: Einaudi.

Weinrich, Harald (1993): Textgrammatik der deutschen Sprache. Mannheim: Dudenverlag.

Veldre-Gerber, Georgia (2012): Demonstrativa im Text. Berlin / New York: de Gruyter.

Zifonun, Gisela / Hoffmann, Ludger / Strecker, Bruno et al. (1997): Grammatik der deutschen Sprache. 3 Bde. Berlin / New York: de Gruyter.

Zifonun, Gisela (2005): Grammatik des Deutschen im europäischen Vergleich. Das Pronomen. Teil III: Possessivpronomen. amades 3. Mannheim: IDS. 